Research Article

\title{
Degenerate Four-Wave Mixing in Phycoerythrin Dye-Doped Nanoparticles
}

\author{
Tomoki Tsuchiya $(\mathbb{D}$ and Chikara Egami \\ Faculty of Engineering, Shizuoka University, 3-5-1 Johoku, Naka-ku, Hamamatsu 432-8561, Japan \\ Correspondence should be addressed to Tomoki Tsuchiya; tsuchitomo278@gmail.com
}

Received 22 February 2021; Accepted 10 June 2021; Published 18 June 2021

Academic Editor: Stefan Wabnitz

Copyright ( 92021 Tomoki Tsuchiya and Chikara Egami. This is an open access article distributed under the Creative Commons Attribution License, which permits unrestricted use, distribution, and reproduction in any medium, provided the original work is properly cited.

We have generated a phase-conjugate (PC) wave from nanoparticles with a new microscopic system proposed. The microscope includes a confocal system with a degenerate four-wave mixing (DFWM) system, which plays a major role in generating the phase-conjugate wave to compensate phase distortion in the optical path toward targets. The proposed optical system detects feeble PC wave and imagines 3D particles while improving the inplane contrast resolution of the microscopic image.

\section{Introduction}

Organic days have attracted extensive attention in optical memory, optical communications, optical information processing, OPC, holographic recording, third harmonic generation (THG), stimulated scattering, and so on [1-8]. In 1972, Zel'dovich et al. first clarified the concept of the phaseconjugate (PC) wave. It has the ability to perform measurements with high accuracy by the phase correction of phase-conjugate light. After that, the degenerate four-wave mixing (DFWM) system was proposed by Hellwarth as a method to generate the PC wave. This "degenerate" means that all four-wave frequencies are equal. The DFWM system can be measured in all media with no phase shift. Until now, the transport band has been used as a medium for generating the PC wave [9]. In this research, we have succeeded in generating the PC wave from nanoparticles doped with phycoerythrin (PE) dyes for the first time to the best of my belief.

In this paper, we target on exploiting a novel microscope, which includes the confocal optical system with the DFWM system. The purpose is to increase the maximum value of the scattering signal by generating the $\mathrm{PC}$ wave and to improve the resultant inplane contrast resolution. Lots of conventional microscopic systems use confocal optical microscopy for cell observation. However, the inplane resolution of the confocal system is determined by the wavelength of a laser and the numerical aperture (NA) of the objective lens. Therefore, we have an idea for using the PC wave exhibiting nonlinear optical response to improve the inplane contrast resolution in the confocal optical system. We evaluated the characteristics of the phase-conjugate wave for the microscopic optical system and performed 3D measurement using organic dye-doped nanoparticles. We used nanoparticles doped with PE dyes as a nonlinear optical material for PC wave generation. The $\mathrm{PE}$ dye is a pigment protein present in red algae and has a large absorption in the wavelength of our light source (see Figure 1).

In our paper, we employed the combined four-wave mixing optical system with the confocal optical system. The proposed confocal optical system is designed so that the focal point of that and the focal point of the imaging lens have a confocal relationship as its name suggests. When there is a target at the focal position of the objective lens, the scattered signal passes through the pinhole at the focal point of the imaging lens. When the target is in a position deviated from the focal point of the objective lens, the scattered signal is not imaged at the focal position of the imaging lens, and most of the signal is blocked by the pinhole. Therefore, by using the confocal optical system, it is possible to detect only 


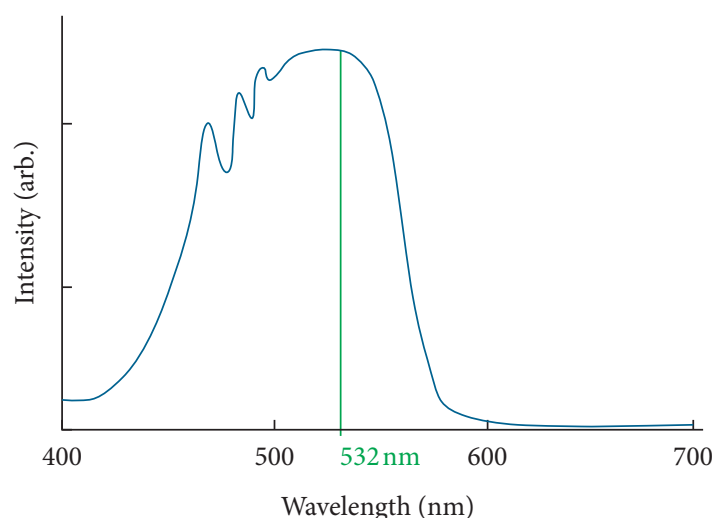

Figure 1: Absorption spectrum of PE-dye-doped polystyrene nanoparticle.

the information at the focal position at the objective lens [10]. Furthermore, while moving the piezo-stage on which the sample is placed, we can get a 3D image converted into digital data.

The DFWM system is an optical configuration for generating the PC wave. The DFWM system uses 3 beams: probe beam $\left(E_{\mathrm{pr}}\right)$, forward pump beam $\left(E_{\mathrm{f}}\right)$, and backward pump beam $\left(E_{\mathrm{b}}\right)$ to generate the PC wave. At this time, the total incident electric field $E$ is written as

$$
E=E_{\mathrm{f}}+E_{\mathrm{b}}+E_{\mathrm{pr}} \text {. }
$$

Now, a light wave generated from the nonlinear medium $\left(E_{\mathrm{PC}}\right)$ is omitted for simplicity. The third-order nonlinear electrical polarization contributing to generation of the PC wave is expressed as follows:

$$
\left.P_{\mathrm{pc}}^{(3)}(r, t)=\frac{3}{2} \varepsilon_{0} \chi^{(3)} E_{f} E_{b} E_{\mathrm{pr}}^{*} \exp \left[i\left\{\left(k_{\mathrm{f}}+k_{\mathrm{b}}-k_{\mathrm{pr}}\right) \cdot r-\omega t\right)\right\}\right] .
$$

Now, $\varepsilon_{0}$ is the dielectric constant in vacuum and $\chi^{(3)}$ is the tensor component of the third-order nonlinear susceptibility. In this paper, we consider the degeneracy case. For this reason, the frequency of $P_{\mathrm{pc}}^{(3)}$ is also the same $\omega$. The wave number $P_{\mathrm{pc}}^{(3)}$ is written as

$$
k_{\mathrm{pc}}=k_{\mathrm{f}}+k_{\mathrm{b}}-k_{\mathrm{pr}} \text {. }
$$

Since two pump beams are incident opposite to each other, $k_{\mathrm{f}}=-k_{\mathrm{b}}$. That is to say, $k_{\mathrm{pc}}=-k_{\mathrm{pr}}$, which indicates that the phase matching condition is satisfied. Therefore, in the DFWM system, if two pump beams are incident opposite to each other, the phase matching condition is automatically satisfied and the PC wave is generated.

The confocal signal is generated from the area within the focal depth of the objective, which determines the corresponding effective wave-mixing interaction length for PC generation in the confocal DFWM system proposed. The interaction length is estimated as $283 \mu \mathrm{m}$ for $\mathrm{NA}=0.9$. It is also well known that the PC wave diffraction efficiency increases with increasing the product of absorption coefficient $\left(\alpha_{0}\right)$ and film thickness (L) at an incident beam intensity high above the saturation intensity $\left(\mathrm{I}_{\text {sat }}\right)$ of resonant absorptive materials. Our confocal DFWM system regards the interaction length corresponding to the film thickness $\mathrm{L}$ for the usual PC generation as constant in focal depth. Therefore, if the focused incident beam intensity is constant within the objective's focus depth, the confocal PC wave linearly depends on $\alpha_{0}$, which means absorptive focus depth, which means absorptive molecular density. Furthermore, the confocal microscope installed in DFWM sensitively detects a small portion of the PC wave generated. In practice, therefore, the very low PC wave diffraction efficiency should never be a problem. As a result, the confocal DFWM system three dimensionally measures the PC wave which imagines the fine structure of resonant absorptive materials.

Generating the PC wave is derived from the third-order nonlinear optical effect. Since all materials have third-order nonlinear susceptibility causing the effect, the phase-conjugate wave generation is also possible in all media. However, since there is a limit to the output power of lasers in practice, it is limited to a medium having a large nonlinear susceptibility of the nonlinear optical medium. Major nonlinear media are crystals, polymer single crystal, organic compounds, and organic dyes. In this paper, assuming the generation of the PC wave from living cells, we target on organic compounds now. The delocalized conjugate $\pi$ electrons contained in the benzene ring involve the nonlinear susceptibility of organic compounds. In order to obtain large nonlinear susceptibility, it is necessary to use a light source having a wavelength close to the resonant region. In this paper, the PE dye functions as the PC wave generator due to its own saturable absorption properties.

\section{Methods}

Figure 2 shows an experimental setup for the microscopic optical system proposed. The light source of this system is a $\mathrm{YVO}_{4}-\mathrm{SHG}$ laser $(\lambda=532 \mathrm{~nm})$. A high-power coherent light source is essential for pulsed lasers. The proposed DFWM system equipped with confocal microscope can measure with low-power. Therefore, we do not use the pulse laser but use the CW laser [11, 12]. The laser light is modulated at a frequency of $5 \mathrm{kHz}$. This modulation frequency that can remove white noise is sufficiently lower than the response frequency of a sample of the organic material. The scattering signal from the sample, which is converted into an electric signal by the photodiode, passes through the lock-in amplifier. Furthermore, the signal, whose noise is removed by the phase detector in the lock-in amplifier, is recorded as data.

Now, we consider the case of detecting the PC wave involved in the nonlinear susceptibility $\chi_{y x x y}^{(3)}$. A $x$-polarized beam of light from the laser is divided by the beam splitter (BS1) into 2 beams. The diameter of the reflected beam by BS1 is magnified 2.5 times by two plano-convex lenses in order to maximize the numerical aperture (NA) of the objective lens. The expanded beam is divided by BS2. One of the reflected beam by BS2 becomes a probe beam $\left(E_{\mathrm{pr}}\right)$. $E_{\mathrm{pr}}$ is set to be $y$-polarized through a half-wave plate. $E_{\mathrm{pr}}$ is converged on the sample through the objective lens $(\mathrm{NA}=0.9)$. In the ordinary DFWM system, the probe beam 


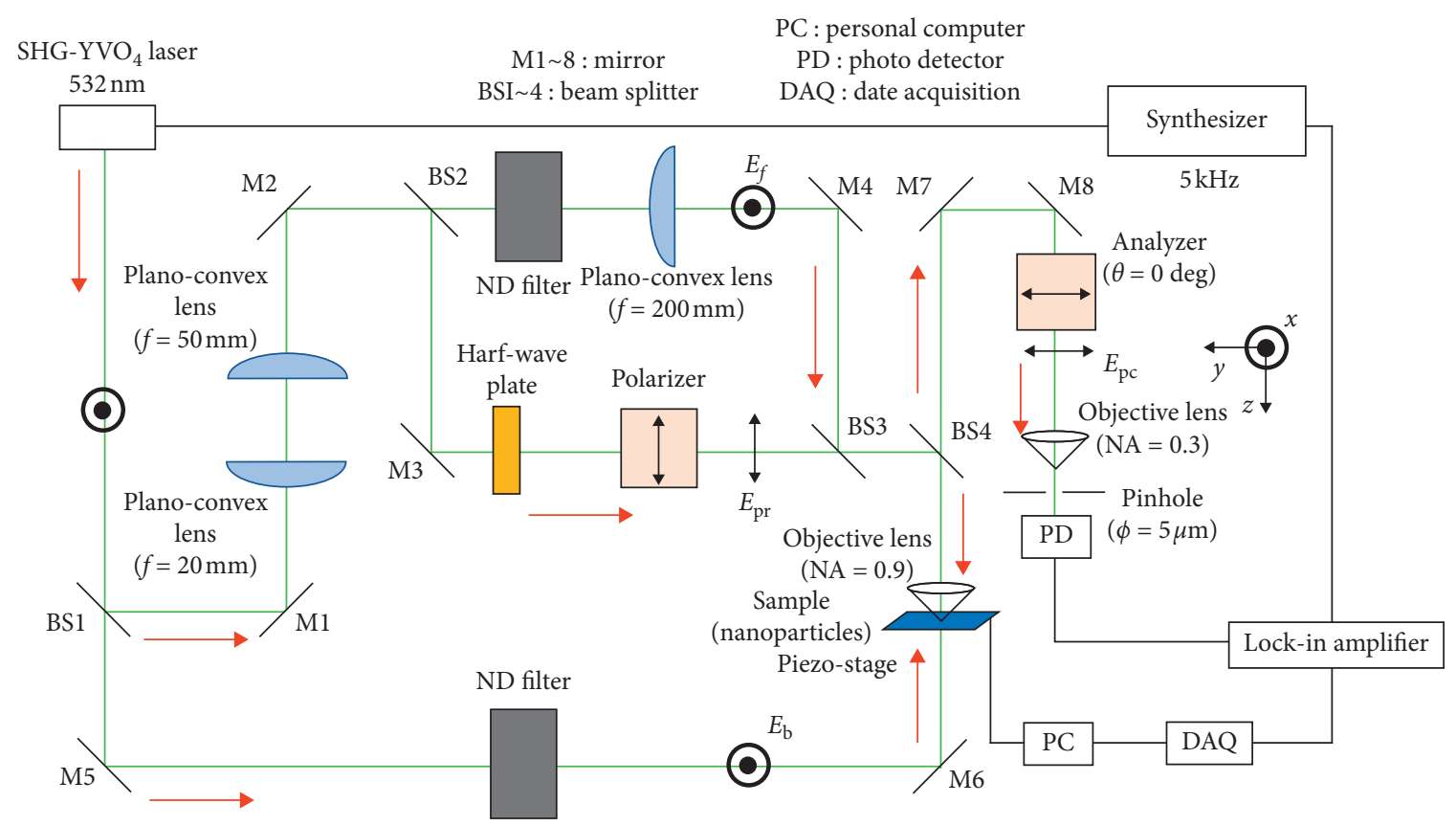

FIGURE 2: Experimental setup for DFWM nonlinear confocal microscope.
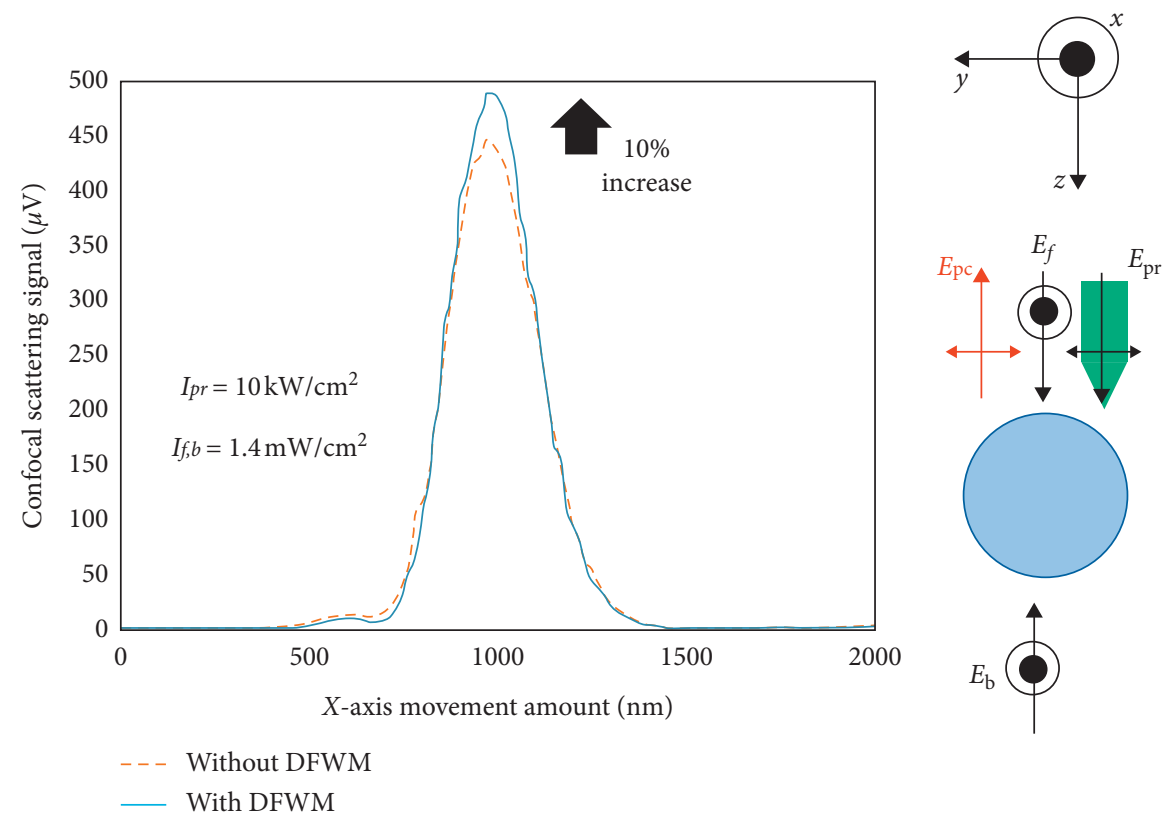

FIgURE 3: Confocal scattering signals from nanoparticle.

has a major role in probing and the pump beams have a major role in exciting and generating $\chi^{(3)}$. In our DFWM system proposed, however, the probe beam has two roles in both probing and exciting. Furthermore, the role of pump beams is to match the mutual phase condition for PC wave generation as well. In this situation, energy flow from pump beams to probe beam is much smaller than that from probe beam to pump beam to pump beams. For that reason, the general DFWM system mostly cannot monitor the PC signal. The proposed DFWM system equipped with a confocal microscope that detects a small part of low scattering can monitor the low PC signal. The general DFWM system measures all interaction areas, but the proposed DFWM system selects and measures only small areas near the focal point. In view of the small interaction region, high dense optical storage by use of dye-doped particles has been reported [13-17].

The other beam by BS2 passing through a plano-convex lens $(f=200 \mathrm{~mm})$ is incident on the sample as a plane beam through an objective lens. On the one hand, this beam 


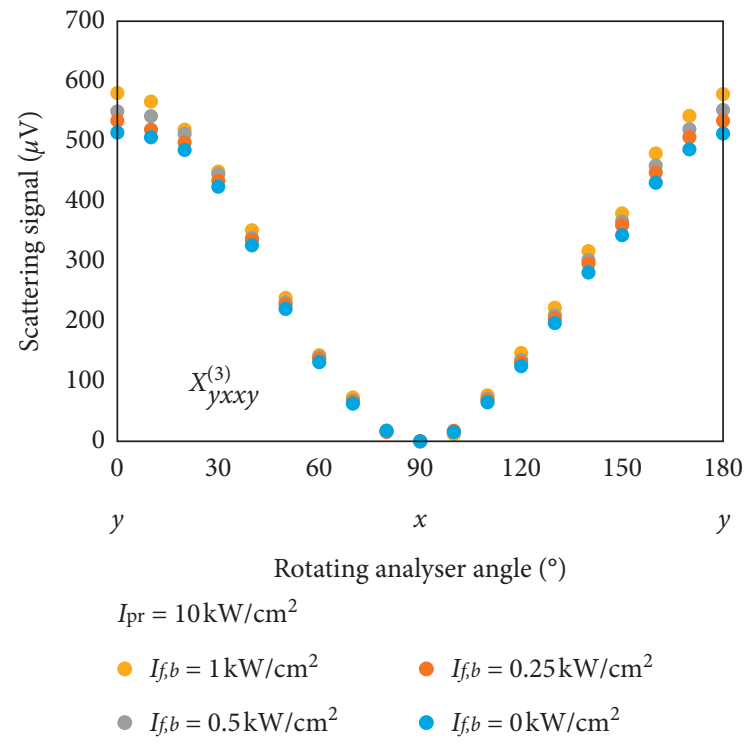

FIgURE 4: Vectorial DFWM beam coupling measured with a confocal microscope.

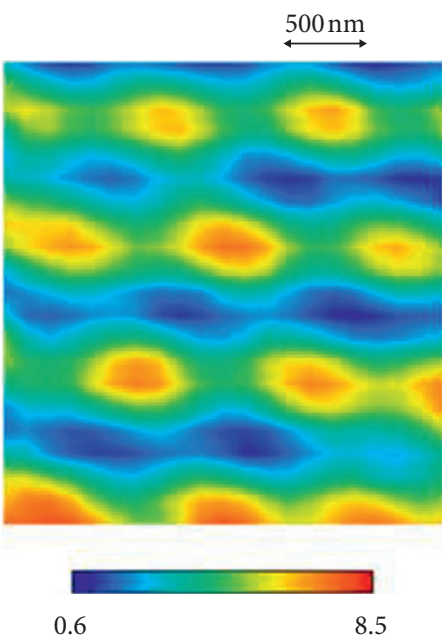

(a)

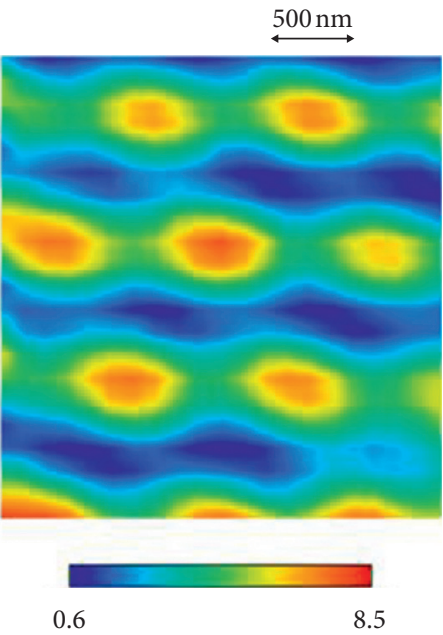

(b)

Figure 5: Tomographic images of two dimensionally arranged PE-dye-doped polystyrene with two types of microscopes: (a) with conventional confocal microscope and (b) with DFWM confocal microscope.

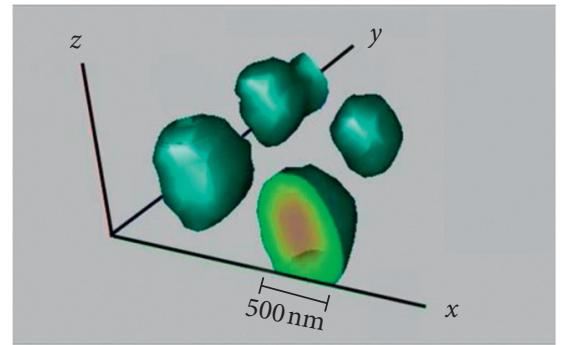

FIGURE 6: 3D image of two dimensionally arranged PE-dye-doped polystyrene nanoparticles with DFWM confocal microscope. 


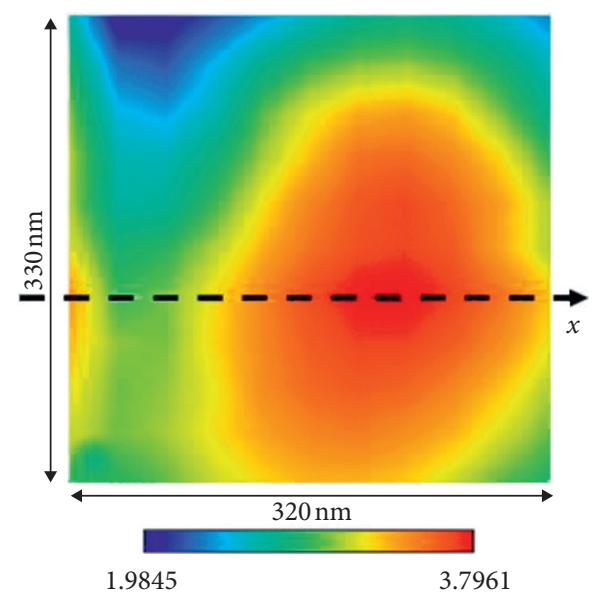

(a)
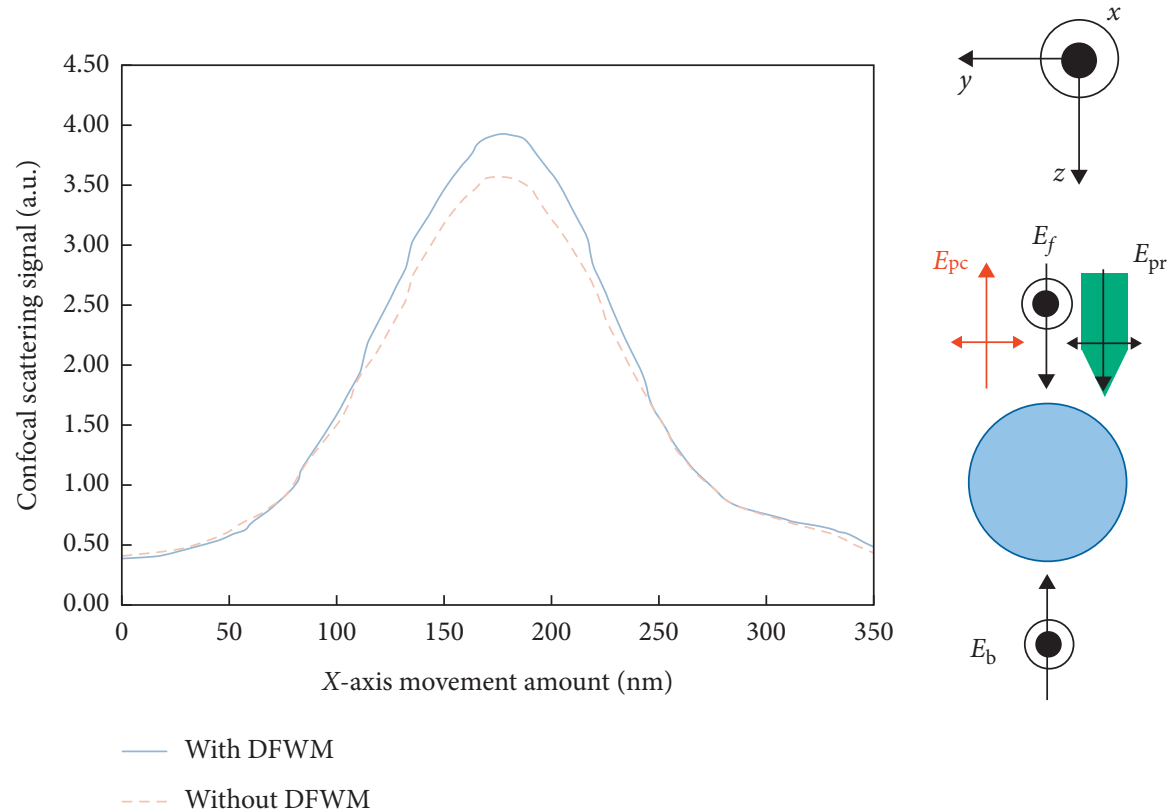

(b)

Figure 7: (a) Tomographic image of two dimensionally arranged PE-dye-doped polystyrene. (b) Confocal scattering signals from nanoparticle with and without DFWM.

becomes $E_{f}$. On the other hand, the beam divided by BS1 is incident on the sample opposite to $E_{\mathrm{pr}}$ and $E_{f}$. This beam becomes counter propagating pump beam, $E_{b}$.

\section{Results and Discussion}

Figure 3 shows the measurement obtained by horizontally scanning the nanoparticle with a diameter of $500 \mathrm{~nm}$ in one direction. The dotted line of Figure 3 is in on the ordinary confocal system, and the solid line is in on the DFWMinstalled confocal system. From Figure 3, you can see more clearly that the increase of the scattering signal by the DFWM confocal system is limited to the vicinity of the central part of the nanoparticle. The scattering signal with the DFWM confocal system is increased by about $10 \%$. At present, the improvement of the in-plane contrast resolution is slight, but from now on, we believe that inplane contrast resolution can be further improved by improving incident light intensities and optical system conditions in the future.

Figure 4 shows the measurement of vectorial DFWM beam coupling with a confocal microscope. $Y$-polarized probe beam $E_{\mathrm{pr}}$ and $x$-polarized counter propagating two pump beams $E_{f, b}$ irradiated a single nanoparticle as a phase conjugator. On the one hand, scattering signal in $y$ polarization at 0 and $180 \mathrm{deg}$ increases with increasing pump beam intensity $I_{f, b}$; on the other hand, scattering signal in $x$ polarization at $90 \mathrm{deg}$ reveals no increase. The experiment confirmed that the $E_{f, b}$ 's beams energies were coupled to 
$E_{\mathrm{pr}}$ 's back scattering energy. The nanoparticle acts as a vectorial phase-conjugate mirror and generates energy coupling by polarization conversion.

Figure 5 shows a 2D centrospherical image of nanoparticles. Figure 5(a) shows the measurement with the usual confocal optical system, and Figure 5(b) shows the measurement with the DFWM system proposed. Comparing Figures 5(a) and 5(b), the maximum color value of the signal in Figure 5(b) is larger than that in Figure 5(a). Furthermore, this is due to the fact that the PC wave is generated by the DFWM system, and the maximum value of the scattering signal is thereby increased. Since the PC wave generates only in the vicinity of the central part of the nanoparticle, that is, in the vicinity of the maximum value of the scattering signal, the in-plane contrast resolution of the image is improved.

Figure 6 shows a 3D image obtained by superimposing the tomographic images shown in Figure 5. You can see 4 nanoparticles with a diameter of $500 \mathrm{~nm}$ in Figure 6 . Looking the cross-sectional view of the nanoparticles in Figure 5, the signal value increases as it is closer to the center. Phase distortion correction effect of DFWM clearly observes the inside of the nanoparticle.

Furthermore, we have succeeded in PC wave generation even in a nanoparticle with a diameter of only $200 \mathrm{~nm}$. Figure 7 (a) shows the measurement with the usual confocal optical system. Figure 7(b) shows the measurement obtained by horizontally scanning the nanoparticle with a diameter of $200 \mathrm{~nm}$ in one direction. The dotted line of Figure 7 is in on the ordinary confocal system, and the solid line is in on the DFWM-installed confocal system. From Figure 7(b), you can see more clearly that the increase of the scattering signal due to polarization vector conversion (energy flow from $x$ polarized $E_{f, b}$ to $y$-polarized $E_{\mathrm{pr}}$ ) by the DFWM confocal system is limited to the vicinity of the central part of the nanoparticle as with Figure 3.

\section{Conclusions}

We have succeeded in generating the PC wave from nanoparticles doped with PE dyes by combining the DFWM system with the confocal optical system for the first time. By superimposing these image data, we have succeeded in creating a high-contrast 3D image. The proposed optical system has a potential to improve the inplane contrast resolution of the nanoparticle's image by the phase-conjugate wave.

\section{Data Availability}

The data used to support the findings of this study are included within the article.

\section{Conflicts of Interest}

The authors declare that they have no conflicts of interest. emission from $\pi$-conjugated dye-doped thin film," Optical Review, vol. 13, no. 4, pp. 239-241, 2006.

[2] V. P. Pham, T. Galstyan, A. Granger, and R. A. Lessard, "Novel azo dye-doped poly (methyl methacrylate) films as optical data storage media," Japanese Journal of Applied Physics, vol. 36, no. 1B, pp. 429-438, 1997.

[3] H. Fujiwara, T. Takeda, and K. Nakagawa, "Organic dyedoped polymer films for optical phase-conjugate processing," Optical Review, vol. 1, no. 2, pp. 243-245, 1994.

[4] C. Egami, K. Nakagawa, and H. Fujiwara, "Efficient optical phase conjugation in methyl-orange-doped polyvinyl alcohol film," Japanese Journal of Applied Physics, vol. 31, no. 9A, pp. 2937-2940, 1992.

[5] M. Ivanov and T. Eiju, "Azodye gelatin films for polarization holographic recording," Optical Review, vol. 8, no. 5, pp. 315-317, 2001.

[6] R. A. Ganeev, S. R. Kamalov, M. K. Kodirov et al., "Harmonic generation in organic dye vapors," Optics Communications, vol. 184, no. 1-4, pp. 305-308, 2000.

[7] E. M. Gromov and B. A. Malomed, "Damped solitons in an extended nonlinear Schrödinger equation with a spatial stimulated Raman scattering and decreasing dispersion," Optics Communications, vol. 320, pp. 88-93, 2014.

[8] E. Nitanai and S. Miyanaga, "Measurements of dispersion properties of refractive indices and absorption coefficients in organic-dye-doped thin films by a prism-coupling method," Optical Engineering, vol. 35, no. 3, p. 900, 1996.

[9] J. Sakai, Phase Conjugation Optics, Asakura Shoten, Tokyo, Japan, 2nd edition, 1996.

[10] D. S. Simon and A. V. Sergienko, "The correlation confocal microscope," Optics Express, vol. 18, no. 10, p. 9765, 2010.

[11] H. Tanaka, A. Horikoshi, H. Fujiwara, and K. Nakagawa, "Phase conjugation in saturable absorbing dye films by degenerate four-wave mixing and holographic processes using nanosecond pulse and CW lasers," Optical Review, vol. 9, no. 3, pp. 106-111, 2002.

[12] A. N. Simonov, A. V. Larichev, V. P. Shibaev, and A. I. Stakhanov, "High-quality correction of wavefront distortions using low-power phase conjugation in azo dye containing LC polymer," Optics Communications, vol. 197, no. 1-3, pp. 175-185, 2001.

[13] T. Geethakrishnan, P. Sakthivel, and P. K. Palanisamy, "Triphenylmethane dye-doped gelatin films for low-power optical phase-conjugation," Optics Communications, vol. 335, pp. 218-223, 2015.

[14] C. Egami, K. Nakagawa, and H. Fujiwara, "Phase conjugation in methyl-orange-doped PVA films by photoinduced anisotropy," Japanese Journal of Applied Physics, vol. 29, p. 1544, 1990.

[15] T. Sato, K. Nakagawa, and H. Fujiwara, "Polarization-dependent light-gated phase conjugation in methyl-orange doped polymer film," Optical Review, vol. 9, no. 4, pp. 154-158, 2002.

[16] A. Tomioka, S. Kinosita, Y. Itakura, and T. Motokubota, "Near-field and polarized evanescent field excited fluorescence of self-organized particle arrays of organic dye," Optical Review, vol. 13, no. 4, pp. 297-301, 2006.

[17] N. Kobayashi and C. Egami, "High-resolution optical storage by use of minute spheres," Optics Letters, vol. 30, no. 3, p. 299, 2005.

\section{References}

[1] Y. Itakura, A. Tomikam, S. Kinosita, and T. Motokubota, "Photoluminescence and possible amplified spontaneous 